\title{
Quatre fois vingt
}

\section{Bernhard Gurtner}

Dr méd., ancien médecin chef de l'Hôpital de Wetzikon, membre de la FMH

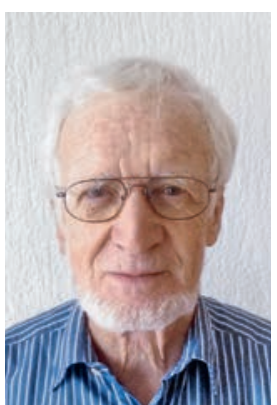

Les Français ont une façon flatteuse de se rajeunir quand ils fêtent leur quatre-vingtième anniversaire: quatre fois vingt. Nos voisins francophones enjolivent pareillement leur soixante-dixième anniversaire. Nos séniors de Suisse romande sont moins hypocrites quand ils reconnaissent porter déjà leur «septante» ans.

Ce «quatre-vingt» remonte au temps où Obelix et ses amis gaulois ne pouvaient compter que jusqu'à vingt, et avaient besoin pour cela de tous leurs doigts, des pieds et des mains. Ils arrivaient à vingt avec les deux mains et les deux pieds, et à 80 quand ils se mettaient à quatre. Quatre fois vingt.

Si 25 octogénaires se retrouvent pour une photo de classe avec leurs anciens camarades, ils cumulent à eux seuls 2000 ans d'expérience de vie, deux millénaires post christum natum. Une année représente pour eux 1,25\% de leur temps de vie. Leur petit-fils de quatre ans en revanche doit attendre un quart de la durée de son existence avant de pouvoir souffler ses bougies d'anniversaire. C'est pourquoi les plus jeunes

\section{Tout le monde aimerait pouvoir remettre les compteurs à zéro, comme le compteur kilo- métrique d'une voiture de collection.}

trouvent infiniment long de patienter jusqu'à leur prochain anniversaire, alors qu'au bout de quelques mois, leurs grands-parents ont la sensation que c'était hier qu'ils fêtaient un an de plus. Leurs oreilles et leurs yeux fatigués leur rappellent que les jeunes gens parlent beaucoup trop vite et trop bas et que les oiseaux volent de plus en plus vite...

Tout le monde aimerait pouvoir remettre les compteurs à zéro, comme le compteur kilométrique d'une voiture de collection. On peut maintenant enregistrer toutes les révisions (articulation de la hanche, stent, cataracte, implants dentaires) et autres données sur la santé sur une puce implantée sous la peau. «Se faire trouer la peau" prend désormais une tout autre signifi- cation. Nous n'en sommes pas encore à nous faire tatouer un code $\mathrm{QR}$ derrière l'oreille gauche avec notre date de péremption personnelle.

Impossible de planter 80 bougies sur un gâteau d'anniversaire, même si elles étaient aussi fines que des cure-dents. Comme au mikado, on pourrait utiliser des bougies de différentes couleurs pour chaque dizaine, le rouge pour 50 , le bleu pour 20 , le vert pour 10 et le jaune pour 5 . Ou mettre une seule bougie très épaisse, avec des anneaux symbolisant les années, et des épingles ou des petits fanions colorés, plantés dessus pour marquer les dates importantes dans la biographie du héros de la fête.

En conduisant, les séniors entraînent tous les jours leur cerveau et entretiennent leurs réflexes. Même parmi les personnes les plus âgées, beaucoup n'ont pas besoin d'être assistées, du moins au volant, d'autant que dans les voitures modernes de nombreuses fonctions sont automatisées. Depuis le siècle dernier déjà, le pied gauche n'est plus nécessaire pour embrayer ou débrayer. Cela fait bien longtemps que la direction assistée, l'aide au stationnement, le régulateur de vitesse et le GPS sont passés dans les habitudes. Et bientôt, les voitures autonomes.

Visiblement il n'y a plus que notre carcasse qui se comporte comme une vieille bicyclette rouillée, nous permettant de faire encore quelques tours de piste sur une selle grinçante, pourvu qu'on l'ait graissée tous les jours et qu'on ait remplacé beaucoup de pièces. Ceux qui s'entraînent régulièrement pour se maintenir en forme physiquement et intellectuellement n'ont qu'à s'en prendre à eux-mêmes si, dans les transports en communs bondés, les jeunes filles collées à leur téléphone portable leur donnent vingt ans de moins et ne leur laissent pas de place assise. Quatre fois vingt!

Espérons qu'au temps de jeu supplémentaire on nous sanctionnera le plus tard possible par un carton jaune ou un carton rouge. Happy Birthday! 